\title{
Numerical Approximation of Real Finite Nonnegative Function by the Modulus of Discrete Fourier Transform
}

\author{
Petro Savenko, Myroslava Tkach \\ Pidstryhach Institute for Applied Problems of Mechanics and Mathematics, National Academy of Sciences of Ukraine, \\ Lviv, Ukraine \\ Email: $\{$ spo,tmd\}@iapmm.lviv.ua \\ Received March 15, 2010; revised April 21, 2010; accepted April 28, 2010
}

\begin{abstract}
The numerical algorithms for finding the lines of branching and branching-off solutions of nonlinear problem on mean-square approximation of a real finite nonnegative function with respect to two variables by the modulus of double discrete Fourier transform dependent on two parameters, are constructed and justified.
\end{abstract}

Keywords: Mean-Square Approximation, Discrete Fourier Transform, Two-Dimensional Nonlinear Integral Equation, Nonuniqueness And Branching of Solutions.

\section{Introduction}

The mean-square approximation of real finite nonnegative function with respect to two variables by the modulus of double discrete Fourier transform dependent on physical parameters, is widely used, in particular, at modeling and solution of the synthesis problems of different types of antenna arrays, signal processing etc. [1-3]. Nonuniqueness and branching of solutions are essential features of nonlinear approximation problem which remains unexplored. The problem on finding the set of branching points, in turn, is not adequately explored nonlinear spectral two-parametric problem. The methods of investigation and numerical finding the solutions of one-parametric spectral problems at presence of discrete spectrum [4-8] are most well-developed. The existence of coherent components of spectrum, which are spectral lines for the case of real parameters [9], is essential difference of nonlinear two-parametric spectral problems.

In the work a variational problem on the best meansquare approximation of a real finite nonnegative function by the module of double discrete Fourier transform is reduced to finding the solutions of Hammerstein type nonlinear two-dimensional integral equation. Using the Schauder principle the existence of solutions is proved. The existence theorem of coherent components of spectrum of holomorphic matrix functions dependent on two spectral parameters is proved. It justifies the application of implicit functions methods to multiparametric spectral problems [9]. The applicability of this theorem to the analysis of spectrum of two-dimensional integral homogeneous equation to which is reduced the problem on finding the lines of possible branching of solutions of the Hammerstein equation, is shown. Algorithms for numerical finding the optimum solutions of an approximation problem are constructed and justified. Numerical examples are presented.

\section{Problem Formulation, Basic Equations and Relations}

Consider the special case of double discrete Fourier transform

$$
f\left(s_{1}, s_{2}\right)=\sum_{n=-N_{1}}^{N_{2}} \sum_{m=-M_{1}(n)}^{M_{2}(n)} I_{n m} \exp \left[i\left(\& / \alpha_{n m} s_{1}+\& \mathscr{C}_{2} y_{n m} s_{2}\right)\right]
$$

setting here $x_{n m}=n \Delta_{x} \quad(n=-N \div N), \quad y_{n m}=m \Delta_{y}$ $(m=-M \div M) ; \quad c_{1}=8 / \Delta_{x}, \quad c_{2}=8 / 2 \Delta_{y}$. If it is necessary for the accepted assumptions we shall consider the formula

$$
f\left(s_{1}, s_{2}\right)=A \mathbf{I} \equiv \sum_{n=-N}^{N} \sum_{m=-M}^{M} I_{n m} \exp \left[i\left(c_{1} n s_{1}+c_{2} m s_{2}\right)\right]
$$

as a linear operator, acting from complex finite-dimensional space $H_{I}=£^{N_{2} \times M_{2}}\left(N_{2}=2 N+1, M_{2}=2 M+1\right)$ into the space of complex-valued continuous functions with respect to two real variables determined in the domain

$$
\Omega=\left\{\left(s_{1}, s_{2}\right):\left|s_{1}\right| \leq \pi / c_{1},\left|s_{2}\right| \leq \pi / c_{2}\right\} .
$$


Here $c_{1}, c_{2}$ are any real non-dimensional numerical parameters belonging to

$$
\Lambda_{c}=\left\{\left(c_{1}, c_{2}\right): 0<c_{1} \leq a, 0<c_{2} \leq b\right\} .
$$

The function $f\left(s_{1}, s_{2}\right)$ is $2 \pi / c_{1}$ - periodic function on argument $s_{1}$ and $2 \pi / c_{2}$ - periodic on $s_{2}$.

In considered spaces we introduce scalar products and generable by them norms

$$
\begin{gathered}
\left(\mathbf{I}_{1}, \mathbf{I}_{2}\right)_{H_{I}}=\frac{4 \pi^{2}}{c_{1} c_{2}} \sum_{n=-N}^{N} \sum_{m=-M}^{M} I_{n m} \overline{I_{n m}}, \\
\|\mathbf{I}\|=(\mathbf{I}, \mathbf{I})_{H_{I}}^{1 / 2}, \\
\left(f_{1}, f_{2}\right)_{C_{(\Omega)}^{(2)}}=\iint_{\Omega} f_{1}\left(s_{1}, s_{2}\right) \overline{f_{2}\left(s_{1}, s_{2}\right)} d s_{1} d s_{2}, \\
\|f\|=(f, f)_{C_{(\Omega)}^{(2)}}^{1 / 2} .
\end{gathered}
$$

Denote an augmented space of continuous functions with entered scalar product and norm (3) as $\mathbf{C}_{(\Omega)}^{(2)}$ and notice that its augmentation coincides with the Hilbert space $L_{2}(\Omega) \quad[10]$.

By direct check we are sure that such equality

$$
\|A \mathbf{I}\|^{2}=\iint_{\Omega}\left|f\left(s_{1}, s_{2}\right)\right|^{2} d s_{1} d s_{2}=\sum_{n, m}\left|I_{n m}\right|^{2}=\|\mathbf{I}\|^{2}
$$

is valid. From here follows, that $A$ is isometric operator in sense (4).

Using the entered scalar products (2) and (3) we find the conjugate operator required later on

$$
\begin{gathered}
A^{*} f=\frac{c_{1} c_{2}}{4 \pi^{2}} \iint_{\Omega} f\left(s_{1}, s_{2}\right) \exp \left[-i\left(c_{1} n s_{1}+c_{2} m s_{2}\right)\right] d s_{1} d s_{2} \\
(n=-N \div N, m=-M \div M) .
\end{gathered}
$$

Let such function be given

$$
\left.P / \rho_{s_{1}}, s_{2}\right)= \begin{cases}F\left(s_{1}, s_{2}\right), & \left(s_{1}, s_{2}\right) \in \bar{G} \subseteq \Omega, \\ 0, & \left(s_{1}, s_{2}\right) \in \Omega \backslash \bar{G},\end{cases}
$$

where $F\left(s_{1}, s_{2}\right)$ is a real continuous and nonnegative in the domain $\bar{G}$ function.

Consider a problem on the best mean-square approximation of the function $F\left(s_{1}, s_{2}\right)$ in the domain $\Omega$ by the module of double discrete Fourier transform (1) owing to select of coefficients of the vector I. We shall formulate it as a minimization problem of the functional

$$
\sigma(\mathbf{I})=\left\|F-\left|A \mathbf{I}\left\|_{\mathbf{C}_{(\Omega)}^{(2)}}^{2} \equiv\right\| F-\right| f \mid\right\|_{\mathbf{C}_{(\Omega)}^{(2)}}^{2}
$$

in the Hilbertian space $H_{I}$. Taking into account (4) and (5), we write the functional $\sigma(\mathbf{I})$ in a simplified form

$$
\sigma(\mathbf{I})=\|F\|_{\mathbf{c}_{(\Omega)}^{(2)}}^{2}-2(F,|A \mathbf{I}|)_{\mathbf{c}_{(\Omega)}^{(2)}}^{2}+\|\mathbf{I}\|_{H_{I}}^{2} .
$$

On the basis of necessary condition of functional minimum we obtain a nonlinear system of equations relating to the components of vector $\mathbf{I}$ in the space $H_{I}$ that are represented in the vector and expanded forms, respectively:

$$
\begin{gathered}
\mathbf{I}=A^{*}\{F \exp [i \arg (A \mathbf{I})]\}, \\
I_{n m}=\frac{c_{1} c_{2}}{4 \pi^{2}} \iint_{\Omega} F\left(s_{1}, s_{2}\right) \exp \left\{i \left[\operatorname { a r g } \left(\sum_{k=-N}^{N} \sum_{l=-M}^{M} I_{n m} \times\right.\right.\right. \\
\left.\left.\left.\times \exp \left[i\left(c_{1} k s_{1}+c_{2} l s_{2}\right)\right]\right)-\left(c_{1} n s_{1}+c_{2} m s_{2}\right)\right]\right\} d s_{1} d s_{2} \\
(n=-N \div N, m=-M \div M) .
\end{gathered}
$$

Acting on both parts of (9) by operator $A$ we obtain equivalent to (9) the Hammerstein type nonlinear integral equation relating to $f$ :

$$
f(Q)=\mathbf{B} f \equiv \iint_{\Omega} K\left(Q, Q^{\prime}, \mathbf{c}\right) F\left(Q^{\prime}\right) \exp \left[i \arg f\left(Q^{\prime}\right)\right] d Q^{\prime},
$$

where $Q^{\prime}=\left(s_{1}^{\prime}, s_{2}^{\prime}\right), d Q^{\prime}=d s_{1}^{\prime} d s_{2}^{\prime}, \mathbf{c}=\left(c_{1}, c_{2}\right)$;

$$
\begin{gathered}
K\left(Q, Q^{\prime}, \mathbf{c}\right)=K_{1}\left(s_{1}, s_{1}^{\prime}, c_{1}\right) \cdot K_{2}\left(s_{2}, s_{2}^{\prime}, c_{2}\right), \\
K_{1}\left(s_{1}, s_{1}^{\prime}, c_{1}\right)=\frac{c_{1}}{2 \pi} \sum_{n=-N}^{N} \exp \left[i c_{1} n\left(s_{1}-s_{1}^{\prime}\right)\right] \equiv \\
\equiv \frac{c_{1}}{2 \pi} \frac{\sin \left[\frac{N_{2} c_{1}}{2}\left(s_{1}-s_{1}^{\prime}\right)\right]}{\frac{c_{1}}{2}\left(s_{1}-s_{1}^{\prime}\right)}, \\
K_{2}\left(s_{2}, s_{2}^{\prime}, c_{2}\right)=\frac{c_{2}}{2 \pi} \frac{\sum_{m=-M}^{M} \exp \left[i c_{2} m\left(s_{2}-s_{2}^{\prime}\right)\right] \equiv}{\equiv \frac{c_{2}}{2 \pi} \frac{\sin \left[\frac{M_{2} c_{2}}{2}\left(s_{2}-s_{2}^{\prime}\right)\right]}{\frac{c_{2}}{2}\left(s_{2}-s_{2}^{\prime}\right)} .}
\end{gathered}
$$

Note, that the kernel (11) of Equation (10) is degenerate and real.

We shall consider one of the properties of function $\exp \left(i \arg f\left(Q^{\prime}\right)\right)$ entering into (10) at $f\left(Q^{\prime}\right) \rightarrow 0$. Obviously that the function

$$
\exp \left(i \arg f\left(Q^{\prime}\right)\right)=\frac{f\left(Q^{\prime}\right)}{\left|f\left(Q^{\prime}\right)\right|} \equiv \frac{u\left(Q^{\prime}\right)+i v\left(Q^{\prime}\right)}{\left(u^{2}\left(Q^{\prime}\right)+v^{2}\left(Q^{\prime}\right)\right)^{1 / 2}}
$$

is continuous if $u\left(Q^{\prime}\right)=\operatorname{Re} f\left(Q^{\prime}\right)$ and $v\left(Q^{\prime}\right)=$ $\operatorname{Im} f\left(Q^{\prime}\right)$ are continuous functions, where $\exp (i \arg f($ $\left.\left.Q^{\prime}\right)\right) \mid=1$ for any $f\left(Q^{\prime}\right)$. If $u\left(Q^{\prime}\right) \rightarrow 0$ and $v\left(Q^{\prime}\right) \rightarrow 0$ simultaneously then $f\left(Q^{\prime}\right) \rightarrow 0$ is a complex zero. Its argument is undetermined accordingly to definition of 
complex zero [11, p. 20]. On this basis we redefine $\exp \left(i \arg f\left(Q^{\prime}\right)\right)$ at $u\left(Q^{\prime}\right) \rightarrow 0$ and $v\left(Q^{\prime}\right) \rightarrow 0$ as a function which has module equal to unit and undetermined argument.

The equivalence of (9) and (10) follows from the following lemma.

Lemma 1. Between solutions of Equations (9) and (10) there exists bijection, i.e., if $\mathbf{I}_{*}$ is a solution of (9) then $f_{*}=A \mathbf{I}_{*}$ is the solution of (10); on the contrary, if $f_{*}$ is the solution of (10) then

$$
\mathbf{I}_{*}=A^{*}\left\{F \exp \left[i \arg \left(f_{*}\right)\right]\right\}
$$

is the solution of (9).

Proof. Let $\mathbf{I}_{*}$ be a solution of (9). Then $\mathbf{I}_{*}-A^{*}$ $\left\{F \exp \left[i \arg \left(A \mathbf{I}_{*}\right)\right]\right\} \equiv 0$. Acting on this identity by the linear operator $A$, we have $A \mathbf{I}_{*}-A A^{*}\{F \exp [i \arg ($ $\left.\left.\left.A \mathbf{I}_{*}\right)\right]\right\} \equiv 0$. Since the operator $A$ acts from the space $H_{I}=\mathrm{f}^{N_{2} \times M_{2}}$ into the space $\mathbf{C}_{(\Omega)}^{(2)}$ and accordingly into the space $H_{f}^{\circ}=L_{2}(\Omega)$, and the set of its nulls consists of only null element from the last identity follows, what $A \mathbf{I}_{*}=f_{*} \in H_{f}^{\circ}$ is a solution of (10).

On the contrary, let $f_{*} \in H_{f}^{\circ}$ solves the Equation (10). The operator $A^{*}$ acts from the space $H_{f}^{o}=L_{2}^{*}(\Omega)$ into the space $H_{I}=\mathrm{f}^{N_{2} \times M_{2}}$ [10] and the Hilbertian space $L_{2}^{*}$ coincides with the space $L_{2}$ [10]. From here follows, that $A^{*}$ acts from the space $H_{f}^{\%}=L_{2}(\Omega)$ into the space $H_{I}=\mathrm{f}^{N_{2} \times M_{2}}$. Taking into account that $F$ is a finite function determined by (6), and $f_{*}$ is continuous, the function $F \exp \left(i \arg \left(f_{*}\right)\right)$ is quadratic integrability in the domain $\Omega$, i.e. $F \exp \left(i \arg \left(f_{*}\right)\right) \in H_{f}$. Thus $A^{*}\left(F \exp \left(i \arg \left(f_{*}\right)\right)\right)=\mathbf{I}_{*} \in H_{I}$ and the right part of (10) is the result of action of operator $A$ on an element $\mathbf{I}_{*}$, i.e. $A \mathbf{I}_{*}=A A^{*}\left(F \exp \left(i \arg \left(f_{*}\right)\right)\right)=f_{*}$. Writing this equality as $A\left(\mathbf{I}_{*}-A^{*}\left(F \exp \left(i \arg \left(A \mathbf{I}_{*}\right)\right)\right)\right)=0$ and taking into account that a set of operator nulls consists of only a null element we obtain $\mathbf{I}_{*}=A^{*}\left(F \exp \left(i \arg \left(A \mathbf{I}_{*}\right)\right)\right)$. So, $\mathbf{I}_{*}=A^{*}(F \exp (i \arg$ $\left.\left.\left(f_{*}\right)\right)\right)$ solves the Equation (9). Lemma is proved.

Thus owing to the equivalence of (9) and (10) we consider simpler of them, namely (10). The Equation (9) is a more complicated equation in sense that in its right part the operator $A$ is in an index of the power of exponent.
Besides taking into account that a set of values of operator $A$ is a set of continuous functions in the domain $\Omega$ belonging to the space $L_{2}(\Omega)$ and this set is a compact in the space $L_{2}(\Omega)$ [12], we shall investigate solutions of (10) in the space $\mathbf{C}(\Omega)$.

Formulate the important properties of (10), which are checked directly.

1) If function $f(Q)$ is a solution of (10) then the conjugate complex function $\overline{f(Q)}$ is also the solution of (10).

2) If function $f(Q)$ is a solution of (10), then $\exp (i \beta) f(Q)$ is also the solution of (10) ( $\beta$ is any real constant).

3) For even on two arguments (or on one argument) functions $F\left(s_{1}, s_{2}\right)$ the nonlinear operator $\mathbf{B}$ that is in the right part of (10), is an invariant concerning the type of parity of the function $\arg f\left(s_{1}, s_{2}\right)$ on two arguments (or on that argument on which $F\left(s_{1}, s_{2}\right)$ is an even function).

Below taking into account the property 2) for uniqueness of solutions we set the parameter $\beta=0$.

Consider the operator

$$
D f \equiv \iint_{\Omega} K\left(Q, Q^{\prime}, \mathbf{c}\right) f\left(Q^{\prime}\right) d Q^{\prime}
$$

and corresponding to it quadratic form

$$
\begin{gathered}
(D f, f)=\iint_{\Omega} \iint_{\Omega} K\left(Q, Q^{\prime}, \mathbf{c}\right) f\left(Q^{\prime}\right) d Q^{\prime} \overline{f(Q)} d Q= \\
=\sum_{n=-N}^{N} \sum_{m=-M}^{M}\left|\iint_{\Omega} \exp \left[i\left(c_{1} n s_{1}+c_{2} m s_{2}\right)\right] \overline{f\left(s_{1}, s_{2}\right)} d s_{1} d s_{2}\right|^{2}= \\
=\left(\frac{2 \pi}{c_{1}}\right)\left(\frac{2 \pi}{c_{2}}\right)\|\mathbf{I}\|^{2} \geq 0 .
\end{gathered}
$$

Obviously that this inequality modifies into equality only as $\mathbf{I}=0$. From here follows that the kernel $K\left(Q, Q^{\prime}, \mathbf{c}\right)$ is positively defined [13]. Accordingly operator $D$ is positive on nonnegative functions cone $\mathrm{K}$ of the space $C(\Omega)$ [14]. According to it $D$ leaves invariant the cone $\mathrm{K}$, i.e. $D \mathrm{~K} \subset \mathrm{K}$.

Complex decomplexified space $\mathbf{C}(\Omega)$ [10] we consider as a direct sum of two real spaces of continuous functions $\mathbf{C}(\Omega)=C(\Omega) \oplus C(\Omega)$ in the domain $\Omega$. The elements of this space are written as $f=(u, v)^{T} \in \mathbf{C}(\Omega)$, $u \in C(\Omega), v \in C(\Omega)$. Norms in these spaces have the form:

$$
\|u\|_{C(\Omega)}=\max _{Q \in \Omega}|u(Q)|, \quad\|v\|_{C(\Omega)}=\max _{Q \in \Omega}|v(Q)|,
$$




$$
\|f\|_{\mathbf{C}(\Omega)}=\max \left(\|u\|_{C(\Omega)},\|v\|_{C(\Omega)}\right) .
$$

The Equation (10) in the decomplexified space $\mathbf{C}(\Omega)$ we reduce to equivalent to it system of the nonlinear equations

$$
\begin{gathered}
u(Q)=B_{1}(u, v) \equiv \iint_{\Omega} K\left(Q, Q^{\prime}, \mathbf{c}\right) F\left(Q^{\prime}\right) \frac{u\left(Q^{\prime}\right)}{\sqrt{u^{2}\left(Q^{\prime}\right)+v^{2}\left(Q^{\prime}\right)}} d Q^{\prime}, \\
v(Q)=B_{2}(u, v) \equiv \iint_{\Omega} K\left(Q, Q^{\prime}, \mathbf{c}\right) F\left(Q^{\prime}\right) \frac{v\left(Q^{\prime}\right)}{\sqrt{u^{2}\left(Q^{\prime}\right)+v^{2}\left(Q^{\prime}\right)}} d Q^{\prime} .
\end{gathered}
$$

Denote the closed convex set of continuous functions as $S_{R} \subset \mathbf{C}(\Omega)$ supposing that

$$
\begin{gathered}
S_{R}=S_{R_{u}} \oplus S_{R_{v}}, S_{R_{u}}=\left\{u:\|u\|_{C(\Omega)} \leq R\right\}, \\
S_{R_{v}}=\left\{v:\|v\|_{C(\Omega)} \leq R\right\}, \\
R=\max _{Q \in \Omega} \iint_{\Omega}\left|K\left(Q, Q^{\prime}, \mathbf{c}\right)\right| F\left(Q^{\prime}\right) d Q^{\prime} .
\end{gathered}
$$

Theorem 1. The operator $\mathbf{B}=\left(B_{1}, B_{2}\right)^{T}$ determined by the Formula (14) maps a closed convex set $S_{R}$ of the Banach space $\mathbf{C}(\Omega)$ in itself and it is completely continuous.

Proof. At first we show that $\mathbf{B}: \mathbf{C}(\Omega) \rightarrow \mathbf{C}(\Omega)$. Let $f=(u, v)^{T}$ be any function belonging to $\mathbf{C}(\Omega)$. At $\left(c_{1}, c_{2}\right) \in \Lambda_{c}$ the kernel $K\left(Q, Q^{\prime}, \mathbf{c}\right)$ is a continuous function with respect to its arguments in the closed domain $\Omega \times \Omega$. Then accordingly to the Cantor theorem [15] $K\left(Q, Q^{\prime}, \mathbf{c}\right)$ is a uniformly continuous function in $\Omega \times \Omega$. From here follows: for any points $\left(Q_{1}, Q_{1}^{\prime}\right)$, $\left(Q_{2}, Q_{2}^{\prime}\right)$ such that whenever $\left|\left(Q_{1}, Q_{1}^{\prime}\right)-\left(Q_{2}, Q_{2}^{\prime}\right)\right|<\delta$, then $\left|K\left(Q_{1}, Q_{1}^{\prime}, \mathbf{c}\right)-K\left(Q_{2}, Q_{2}^{\prime}, \mathbf{c}\right)\right|<\frac{\varepsilon}{a}, \quad$ where $a=$ $\iint_{\Omega} F\left(Q^{\prime}\right) d Q^{\prime}$. On this basis we obtain

$$
\begin{gathered}
\left|u\left(Q_{1}\right)-u\left(Q_{2}\right)\right|=\mid \iint_{\Omega} F\left(Q^{\prime}\right)\left[K\left(Q_{1}, Q^{\prime}, \mathbf{c}\right)-K\left(Q_{2}, Q^{\prime}, \mathbf{c}\right)\right] \times \\
\times \frac{u\left(Q^{\prime}\right)}{\sqrt{u^{2}\left(Q^{\prime}\right)+v^{2}\left(Q^{\prime}\right)}} d Q^{\prime} \mid \leq \frac{\varepsilon}{a} \iint_{G} F\left(Q^{\prime}\right) d Q^{\prime}=\varepsilon, \quad \text { (15) } \\
\text { since } \max _{Q^{\prime} \in \bar{\Omega}}\left|\frac{u\left(Q^{\prime}\right)}{\sqrt{u^{2}\left(Q^{\prime}\right)+v^{2}\left(Q^{\prime}\right)}}\right| \leq 1 .
\end{gathered}
$$

Analogously we have that $\left|v\left(Q_{1}\right)-v\left(Q_{2}\right)\right| \leq \varepsilon$ whenever $\left|\left(Q_{1}, Q_{1}^{\prime}\right)-\left(Q_{2}, Q_{2}^{\prime}\right)\right|<\delta$, i.e. $(u, v)^{T} \in \mathbf{C}(\Omega)$ and
$\mathbf{B}: \mathbf{C}(\Omega) \rightarrow \mathbf{C}(\Omega)$.

To prove the property of a complete continuity of the operator $\mathbf{B}=\left(B_{1}, B_{2}\right)^{T}$ it is necessary to prove its compactness and continuity [12]. Show a continuity $\mathbf{B}=\left(B_{1}, B_{2}\right)^{T}$. Let $f_{1}=\left(u_{1}, v_{1}\right)^{T} \in S_{R}$ be any fixed function and $f_{2}=\left(u_{2}, v_{2}\right)^{T}$ be any function belonging to $S_{R}$. It is necessary to show that $\left\|\mathbf{B} f_{1}-\mathbf{B} f_{2}\right\|_{\mathbf{C}(\Omega)} \rightarrow 0$ as $\left\|f_{1}-f_{2}\right\|_{\mathbf{C}(\Omega)} \rightarrow 0$. Set $u_{2}=u_{1}+\Delta u, \quad v_{2}=v_{1}+\Delta v$. Taking into account these equalities we obtain

$$
\frac{u_{2}}{\sqrt{u_{2}^{2}+v_{2}^{2}}}=\frac{u_{1}+\Delta u}{\sqrt{u_{1}^{2}+v_{1}^{2}} \sqrt{1+\frac{2 u_{1} \Delta u+2 v_{1} \Delta v+\Delta u^{2}+\Delta v^{2}}{u_{1}^{2}+v_{1}^{2}}}} .
$$

At $\|\Delta u\|_{C(\Omega)} \rightarrow 0,\|\Delta v\|_{C(\Omega)} \rightarrow 0$ we have

$$
\begin{aligned}
& \lim _{\substack{\Delta u \\
\Delta \nu \| \rightarrow 0,}}\left\|\frac{u_{1}(Q)}{\sqrt{u_{1}^{2}(Q)+v_{1}^{2}(Q)}}-\frac{u_{2}(Q)}{\sqrt{u_{2}^{2}(Q)+v_{2}^{2}(Q)}}\right\|_{\mathbf{C}(\Omega)} \leq \\
& \leq \lim _{\substack{\Delta u\|\rightarrow 0, \Delta v\| \rightarrow 0}} \max _{Q \in \Omega}\left\{\mid \frac{u_{1}(Q)}{\sqrt{u_{1}^{2}(Q)+v_{1}^{2}(Q)}}\left(1-\frac{1}{P\left(u_{1}(Q), v_{1}(Q)\right)}\right)+\right. \\
& \left.+\left|\frac{\Delta u(Q)}{\sqrt{u_{1}^{2}(Q)+v_{1}^{2}(Q)} P\left(u_{1}(Q), v_{1}(Q)\right)}\right|\right\}=0,
\end{aligned}
$$

where

$$
\begin{gathered}
P\left(u_{1}(Q), v_{1}(Q)\right)= \\
=\sqrt{1+\frac{2 u_{1}(Q) \Delta u(Q)+2 v_{1}(Q) \Delta v(Q)+\Delta u^{2}(Q)+\Delta v^{2}(Q)}{u_{1}^{2}(Q)+v_{1}^{2}(Q)}},
\end{gathered}
$$

since

$$
\lim _{\substack{\Delta u\|\rightarrow 0, \Delta v\| \rightarrow 0}} \max _{Q \in \Omega}\left|P\left(u_{1}(Q), v_{1}(Q)\right)\right|=1 .
$$

Similarly we obtain

$$
\begin{aligned}
& \lim _{\substack{\Delta u\|\rightarrow 0, \Delta v\| \rightarrow 0}} \max _{Q \in \bar{\Omega}}\left|\frac{v_{1}(Q)}{\sqrt{u_{1}^{2}(Q)+v_{1}^{2}(Q)}}-\frac{v_{2}(Q)}{\sqrt{u_{2}^{2}(Q)+v_{2}^{2}(Q)}}\right|=0 . \\
& \text { Thus, from (16) and (17) follows }
\end{aligned}
$$

$$
\begin{aligned}
& \lim _{\substack{\Delta u \mid\|0, \Delta v\| \rightarrow 0}}\left\|B_{1}\left(u_{1}, v_{1}\right)-B_{1}\left(u_{2}, v_{2}\right)\right\|_{\mathbf{C}(\Omega)}= \\
& =\lim _{\substack{\Delta u\|\rightarrow 0, \Delta v\| \rightarrow 0}} \max _{Q \in \bar{\Omega}} \mid \iint_{\Omega} F\left(Q^{\prime}\right) K\left(Q, Q^{\prime}, \mathbf{c}\right) \times
\end{aligned}
$$$$
\times\left(\frac{u_{1}\left(Q^{\prime}\right)}{\sqrt{u_{1}^{2}\left(Q^{\prime}\right)+v_{1}^{2}\left(Q^{\prime}\right)}}-\frac{u_{2}\left(Q^{\prime}\right)}{\sqrt{u_{2}^{2}\left(Q^{\prime}\right)+v_{2}^{2} d Q^{\prime}\left(Q^{\prime}\right)}}\right) d Q^{\prime} \mid=0 \text {. }
$$

Analogously 


$$
\lim _{\substack{\|\Delta u\|_{C(\Omega)} \rightarrow 0, \Delta \Delta v \|_{C(\Omega)} \rightarrow 0}}\left\|B_{2}\left(u_{1}, v_{1}\right)-B_{2}\left(u_{2}, v_{2}\right)\right\|_{\mathbf{C}(\Omega)}=0 .
$$

So, $\mathbf{B}=\left(B_{1}, B_{2}\right)^{T}$ is continuous operator from $\mathbf{C}(\Omega)$ into $\mathbf{C}(\Omega)$.

We show that a set of functions $S_{g}=\mathbf{B} S_{R}$ satisfies conditions of the Arzela theorem [12], i.e. we show that functions of the set $S_{g}$ are uniformly bounded and equipotentially continuous. Furthermore $\mathbf{B} S_{R} \subset S_{R}$. Let $g=(w, \omega)^{T}=\mathbf{B} f \equiv\left(B_{1}(u, v), B_{2}(u, v)\right)^{T}$, where $f=$ $(u, v)^{T}$ is any function of the set $S_{R}$. Then as $\left|\left(Q_{1}, Q^{\prime}\right)-\left(Q_{2}, Q^{\prime}\right)\right|<\delta$ analogously with (15) we have

$$
\left(\begin{array}{l}
\left|w\left(Q_{1}\right)-w\left(Q_{2}\right)\right| \\
\left|\omega\left(Q_{1}\right)-\omega\left(Q_{2}\right)\right|
\end{array}\right) \leq\left(\begin{array}{l}
\frac{\varepsilon}{a} \iint_{\Omega} F\left(Q^{\prime}\right) d Q^{\prime} \\
\frac{\varepsilon}{a} \iint_{\Omega} F\left(Q^{\prime}\right) d Q^{\prime}
\end{array}\right)=\left(\begin{array}{l}
\varepsilon \\
\varepsilon
\end{array}\right) .
$$

Thus functions of the set $S_{g}=\mathbf{B} S_{R}$ are equipotentially continuous.

The uniform boundedness of the set $S_{g}=\mathbf{B} S_{R}$ follows from an inequality

$$
\begin{gathered}
\|g\|_{\mathbf{C}(\Omega)}=\max \left\{\max _{Q \in \Omega} \mid \iint_{\Omega} F\left(Q^{\prime}\right) K\left(Q, Q^{\prime}, \mathbf{c}\right) \times\right. \\
\times \frac{u\left(Q^{\prime}\right)}{\sqrt{u^{2}\left(Q^{\prime}\right)+v^{2}\left(Q^{\prime}\right)}} d Q^{\prime} \mid \leq \\
\left.\leq \max _{Q \in \Omega}\left|\iint_{\Omega} F\left(Q^{\prime}\right) K\left(Q, Q^{\prime}, \mathbf{c}\right) \frac{v\left(Q^{\prime}\right)}{\sqrt{u^{2}\left(Q^{\prime}\right)+v^{2}\left(Q^{\prime}\right)}} d Q^{\prime}\right|\right\} \leq R,
\end{gathered}
$$

where $f=(u, v)^{T}$ is any function of the set $S_{R}$ and $g=\mathbf{B} f \equiv\left(B_{1}(u, v), B_{2}(u, v)\right)^{T}$. From the last inequality we have also $\mathbf{B} S_{R} \subset S_{R}$. So, the operator $\mathbf{B}=\left(B_{1}, B_{2}\right)^{T}$ is completely continuous mapping a closed convex set $S_{R} \subset \mathbf{C}(\Omega)$ into itself.

Theorem is proved.

From the Theorem 1 follows satisfaction of conditions of the Schauder principle [16] according to which the operator $\mathbf{B}=\left(B_{1}, B_{2}\right)^{T}$ has a fixed point $f_{*}=\left(u_{*}, v_{*}\right)^{T}$ belonging to the set $S_{R}$. This point is a solution of a system of Equation (14) and Equation (10), respectively. Substituting $f_{*}=\left(u_{*}, v_{*}\right)^{T}$ into (12), we obtain a solution of (9) being a stationary point of the functional (7).

The solutions of a system of equations analogous with (14) in a case of one-dimensional domains $\Omega$ were investigated for the synthesis problem of linear antenna array in particular in [17]. The obtained there results show that for equations of the type (10) and (14) non-uniqueness and branching of solutions dependent on the size of physical parameter are characteristic. Directly the results [17] cannot be transferred on the two-dimensional two-parametric problem (8) and (14). Here, as unlike the points of branching [17], the branching lines of solutions exist and a problem on finding the lines of branching is a nonlinear two-parametrical spectral problem.

Easily to be convinced that function

$$
f_{0}(Q, \mathbf{c})=\iint_{G} F\left(Q^{\prime}\right) K\left(Q, Q^{\prime}, \mathbf{c}\right) d Q^{\prime}
$$

is one of solutions of (10) in the class of real functions. Since, as shown before, the operator $D$ determined by (13), is positive on the nonnegative functions cone $\mathrm{K} \in \mathrm{C}(\Omega), \quad D \mathrm{~K} \subset \mathrm{K}$ and $F \subset \mathrm{K}$, then $f_{0}=D F$ also is a nonnegative function in the domain $\Omega$.

To find the lines of branching and complex solutions of (10), branching-off from real solution $f_{0}(Q, \mathbf{c})$, we consider a problem on finding such set of values of parameters $\mathbf{c}^{(0)}=\left(c_{1}^{(0)}, c_{2}^{(0)}\right)$ and all distinct from $f_{0}(Q, \mathbf{c})$ solutions of the system (14) which for $\left|\mathbf{c}-\mathbf{c}^{(0)}\right| \rightarrow 0 \quad$ (where $\left.c_{1} \geq c_{1}^{(0)}, \quad c_{2} \geq c_{2}^{(0)}\right)$ satisfy conditions

$$
\max _{Q \in G}\left|u(Q, \mathbf{c})-f\left(Q, \mathbf{c}^{(0)}\right)\right| \rightarrow 0, \max _{Q \in G}|v(Q, \mathbf{c})| \rightarrow 0 \text {. (19) }
$$

These conditions indicate the need to find small continuous in $G$ solutions

$$
w(Q, \mathbf{c})=u(Q, \mathbf{c})-f_{0}\left(Q, \mathbf{c}^{(0)}\right), \omega(Q, \mathbf{c})=v(Q, \mathbf{c}),
$$

which converge uniformly to zero as $\mathbf{c} \rightarrow \mathbf{c}^{(0)}$.

Set

$$
c_{1}=c_{1}^{(0)}+\mu, \quad c_{2}=c_{2}^{(0)}+v
$$

and desired solutions we find in the form

$u(Q, \mathbf{c})=f_{0}\left(Q, \mathbf{c}^{(0)}\right)+w(Q, \mu, \nu), v(Q, \mathbf{c})=\omega(Q, \mu, \nu)$.

Further we omit dependence of the functions $w(Q, \mu, v)$ and $\omega(Q, \mu, v)$ on parameters $\mu$ and $v$.

Notice the properties of integrand in the system (14). They are continuous functions with respect to the arguments. After substitution (20) and (21) into (14) the integrand develop in equiconvergent power series by functional arguments $w$ and $\omega$, numerical parameters $\mu$ and $v$ in the vicinity of a point $\left(\mathbf{c}^{(0)}, f_{0}\left(Q, \mathbf{c}^{(0)}\right), 0\right)$ :

$$
F\left(Q^{\prime}\right) K\left(Q, Q^{\prime}, \mathbf{c}\right) \frac{u\left(Q^{\prime}\right)}{\sqrt{u^{2}\left(Q^{\prime}\right)+v^{2}\left(Q^{\prime}\right)}}=
$$




$$
\begin{gathered}
=\sum_{m+n+p+q \geq 0} A_{m n p q}\left(Q, Q^{\prime}, \mathbf{c}^{(0)}\right) w^{m}\left(Q^{\prime}\right) \omega^{n}\left(Q^{\prime}\right) \mu^{p} v^{q}, \\
F\left(Q^{\prime}\right) K\left(Q, Q^{\prime}, \mathbf{c}\right) \frac{v\left(Q^{\prime}\right)}{\sqrt{u^{2}\left(Q^{\prime}\right)+v^{2}\left(Q^{\prime}\right)}}= \\
=\sum_{m+n+p+q \geq 1} B_{m n p q}\left(Q, Q^{\prime}, \mathbf{c}^{(0)}\right) w^{m}\left(Q^{\prime}\right) \omega^{n}\left(Q^{\prime}\right) \mu^{p} v^{q} .
\end{gathered}
$$

Here $A_{\text {mnpq }}\left(Q, Q^{\prime}, \mathbf{c}^{(0)}\right), B_{\text {mnpq }}\left(Q, Q^{\prime}, \mathbf{c}^{(0)}\right)$ are coefficients of expansion continuously dependent on the arguments. Substituting (20) and (22) into (14) and taking into account that $f_{0}\left(Q^{\prime}, \mathbf{c}^{(0)}\right)$ solves the system (14) we obtain a system of nonlinear equations with respect to small solutions $w, \omega$ :

$$
\begin{gathered}
u(Q)=a_{10}\left(Q, \mathbf{c}^{(0)}\right) \mu+a_{01}\left(Q, \mathbf{c}^{(0)}\right) v+ \\
+\sum_{m+n+p+q \geq 2} \mu^{p} v^{q} \iint_{\Omega} A_{m n p q}\left(Q, Q^{\prime}, \mathbf{c}^{(0)}\right) w^{m}\left(Q^{\prime}\right) \omega^{n}\left(Q^{\prime}\right) d Q^{\prime}, \\
\omega(Q)-\iint_{\Omega} F(Q) K\left(Q, Q^{\prime}, \mathbf{c}^{(0)}\right) \frac{\omega\left(Q^{\prime}\right)}{f_{0}\left(Q^{\prime}, \mathbf{c}^{(0)}\right)} d Q^{\prime}= \\
=\sum_{m+n+p+q \geq 2} \mu^{p} v^{q} \iint_{\Omega} B_{m n p q}\left(Q, Q^{\prime}, \mathbf{c}^{(0)}\right) w^{m}\left(Q^{\prime}\right) \omega^{n}\left(Q^{\prime}\right) d Q^{\prime},
\end{gathered}
$$

where

$$
\begin{aligned}
& a_{10}\left(Q, \mathbf{c}^{(0)}\right)=\iint_{G} A_{0010}\left(Q, Q^{\prime}, \mathbf{c}^{(0)}\right) d Q^{\prime}, \\
& a_{01}\left(Q, \mathbf{c}^{(0)}\right)=\iint_{G} A_{0001}\left(Q, Q^{\prime}, \mathbf{c}^{(0)}\right) d Q^{\prime} .
\end{aligned}
$$

\section{Nonlinear Two-Parametric Spectral Problem}

For further application of methods of the branching theory of solutions of nonlinear equations [18] to a system (23) and (24) it is necessary to find solutions of distinct from trivial of the linear homogeneous integral equation obtained equating to zero the left part of (24)

$$
\varphi(Q)=T\left(c_{1}, c_{2}\right) \varphi \equiv \iint_{G} \frac{F\left(Q^{\prime}\right)}{f_{0}\left(Q^{\prime}, c_{1}, c_{2}\right)} K\left(Q, Q^{\prime}, c_{1}, c_{2}\right) \varphi\left(Q^{\prime}\right) d Q^{\prime}
$$

under condition $f_{0}\left(Q^{\prime}, \mathbf{c}\right)>0$. Indicate that the operator $T(\mathbf{c}): \mathbf{C}(\Omega) \rightarrow \mathbf{C}(\Omega)$ is completely continuous. Proof of this property is similar to the proof of a complete continuity of the operator $\mathbf{B}=\left(B_{1}, B_{1}\right)^{T}$ in the Theorem 1 .

According to [18] such values of parameters $\left(c_{1}^{(0)}, c_{2}^{(0)}\right) \in^{\circ 2}$ at which linear homogeneous Equation (25) has distinct from identical zero solutions are points of possible branching of solutions of a system of nonlinear Equations (23) and (24). The eigenfunctions of (25) are used at construction branching-off solutions of (23) and (24).

The spectral parameters $c_{1}$ and $c_{2}$ are included non- linearly into the kernel of the integral operator. Therefore a problem on finding the distinct from $f_{0}\left(Q, c_{1}, c_{2}\right)$ solutions of (25) is a nonlinear two-parametric spectral problem. It consists in finding such values of real parameters $\left(c_{1}, c_{2}\right) \in \Lambda_{c}$ at which (25) has distinct from identical zero solutions.

In operational form a nonlinear two-parametric problem is presented as

$$
\text { A }\left(c_{1}, c_{2}\right) x \equiv\left(E-T\left(c_{1}, c_{2}\right)\right) x=0 .
$$

Here $E$ is an identical operator and $T\left(c_{1}, c_{2}\right)$ is a linear integrated operator acting in the Banach space $C(\Omega)$. It is necessary to find eigenvalue $\mathbf{c}=\left(c_{1}^{(0)}, c_{2}^{(0)}\right) \in \Lambda_{c}$ and corresponding eigenvectors $x^{(0)} \in C(\Omega)\left(x^{(0)} \neq 0\right)$ such that $\mathrm{A}\left(c_{1}^{(0)}, c_{2}^{(0)}\right) x^{(0)}=0$.

By direct check we ascertain that for any values of parameters $\left(c_{1}, c_{2}\right) \in \Lambda_{c}$ the function

$$
\hat{\varphi}_{0}(Q, \mathbf{c})=\iint_{\Omega} F\left(Q^{\prime}\right) K\left(Q, Q^{\prime}, \mathbf{c}\right) d Q^{\prime}
$$

is one of eigenfunctions.

Write a conjugate to (25) equation required in later

$$
\psi(Q)=T^{*}(\mathbf{c}) \psi \equiv \frac{F(Q)}{f_{0}(Q, \mathbf{c})} \iint_{\Omega} K\left(Q, Q^{\prime}, \mathbf{c}\right) \psi\left(Q^{\prime}\right) d Q^{\prime} .
$$

At arbitrary $\left(c_{1}, c_{2}\right) \in \Lambda_{c}$ the function

$$
\hat{\psi}_{0}(Q)=F(Q) \text {. }
$$

is one of eigenfunctions of (28)

The existence of distinct from identical zero solutions of (25) at arbitrary $\left(c_{1}, c_{2}\right) \in \Lambda_{c}$ testifies to the existence of coherent components of a spectrum conterminous with the domain $\Lambda_{c}$.

For finding the distinct from $\hat{\varphi}_{0}(Q, \mathbf{c})$ solutions we exclude from the kernel of integral Equation (25) the eigen function (27), namely: consider the equation

$$
\varphi(Q, \mathbf{c})=\AA^{\prime}(\mathbf{c}) \varphi \equiv \iint_{\Omega} K\left(Q, Q^{\prime}, \mathbf{c}\right) \varphi\left(Q^{\prime}\right) d Q^{\prime},
$$

where

$$
\begin{aligned}
& \mathrm{K}\left(Q, Q^{\prime}, \mathbf{c}\right)=\frac{F\left(Q^{\prime}\right)}{f_{0}\left(Q^{\prime}, \mathbf{c}\right)} K\left(Q, Q^{\prime}, \mathbf{c}\right)-\Psi_{0}(Q) \varphi_{0}\left(Q^{\prime}, \mathbf{c}\right), \\
& \psi_{0}(Q)=\frac{\hat{\Psi}_{0}(Q)}{\left\|\hat{\Psi}_{0}\right\|_{L_{2}}}, \quad \varphi_{0}\left(Q^{\prime}, \mathbf{c}\right)=\frac{\hat{\varphi}_{0}\left(Q^{\prime}, \mathbf{c}\right)}{\left\|\hat{\varphi}_{0}\right\|_{L_{2}}}
\end{aligned}
$$


From Schmidt Lemma [18] follows that $\varphi_{0}(Q, \mathbf{c})$ will not be an eigenfunction of this equation for any values $\left(c_{1}, c_{2}\right) \in \Lambda_{c}$. Thus from a spectrum of operator there is excluded coherent component coinciding with the domain $\Lambda_{c}$ and corresponding to the function $\varphi_{0}(Q, \mathbf{c})$.

Using the property of degeneration of the kernel $\mathrm{K}\left(Q, Q^{\prime}, c_{1}, c_{2}\right)$, we reduce (25) to equivalent system of linear algebraic equations having coefficients analytically dependent on parameters $c_{1}, c_{2}$. We write (25) as

$$
\begin{gathered}
\varphi\left(s_{1}, s_{2}\right)=\sum_{n=-N}^{N} \sum_{m=-M}^{M} x_{n m} \exp \left[i\left(c_{1} n s_{1}+c_{2} m s_{2}\right)\right]- \\
-x_{0} \psi_{0}\left(s_{1}, s_{2}\right)
\end{gathered}
$$

where $x_{n m}, x_{0}$ are constants determined by the formulas

$$
\begin{gathered}
x_{n m}=\frac{c_{1}}{2 \pi} \frac{c_{2}}{2 \pi} \iint_{\Omega} \frac{F\left(s_{1}^{\prime}, s_{2}^{\prime}\right)}{f_{0}\left(s_{1}^{\prime}, s_{2}^{\prime}, c_{1}, c_{2}\right)} \exp \left[-i\left(c_{1} n s_{1}^{\prime}+c_{2} m s_{2}^{\prime}\right)\right] \times \\
\times \varphi\left(s_{1}^{\prime}, s_{2}^{\prime}\right) d s_{1}^{\prime} d s_{2}^{\prime}(n=-N \div N, m=-M \div M), \\
x_{0}=\iint_{\Omega} \varphi_{0}\left(s_{1}^{\prime}, s_{2}^{\prime}, c_{1}, c_{2}\right) \varphi\left(s_{1}^{\prime}, s_{2}^{\prime}\right) d s_{1}^{\prime} d s_{2}^{\prime} .
\end{gathered}
$$

From the Formula (33) follows, that the function $\varphi\left(s_{1}, s_{2}\right)$ will become known, if will be found $x_{n m}$, $x_{0}$.

Multiplication of both parts of (33) by $\frac{F\left(s_{1}^{\prime}, s_{2}^{\prime}\right)}{f_{0}\left(s_{1}^{\prime}, s_{2}^{\prime}, c_{1}, c_{2}\right)} \exp \left[-i\left(c_{1} k s_{1}^{\prime}+c_{2} l s_{2}^{\prime}\right)\right]$ at $k=-N \div N$, $l=-M \div M$ and by $\varphi_{0}\left(s_{1}^{\prime}, s_{2}^{\prime}\right)$, and integration over $\Omega$ gives a homogeneous system of the linear algebraic equations for finding $x_{n m}, x_{0}$

$$
x_{k l}=\sum_{n=-N}^{N} \sum_{m=-M}^{M} a_{n m}^{(k l)}\left(c_{1}, c_{2}\right) x_{n m}\left(\begin{array}{l}
k=-N \div N, \\
l=-M \div M
\end{array}\right) .
$$

Here

$$
\begin{gathered}
a_{n m}^{(k l)}\left(c_{1}, c_{2}\right)=\left\{t_{n m}^{(k l)}\left(c_{1}, c_{2}\right)-\frac{b^{(k l)}\left(c_{1}, c_{2}\right)}{1+d_{0}\left(c_{1}, c_{2}\right)} d_{n m}\left(c_{1}, c_{2}\right)\right\}, \\
t_{n m}^{(k l)}\left(c_{1}, c_{2}\right)=\frac{c_{1} c_{2}}{4 \pi^{2}} \iint_{\Omega} \frac{F\left(s_{1}, s_{2}\right)}{f_{0}\left(s_{1}, s_{2}, c_{1}, c_{2}\right)} \times \\
\times \exp \left\{i\left[c_{1}(n-k) s_{1}+c_{2}(m-l) s_{2}\right]\right\} d s_{1} d s_{2}, \\
b^{(k l)}\left(c_{1}, c_{2}\right)=\frac{c_{1} c_{2}}{4 \pi^{2}} \iint_{\Omega} \frac{F\left(s_{1}, s_{2}\right)}{f_{0}\left(s_{1}, s_{2}, c_{1}, c_{2}\right)} \psi_{0}\left(s_{1}, s_{2}\right) \times, \\
\times \exp \left[-i\left(c_{1} k s_{1}+c_{2} l s_{2}\right)\right] d s_{1} d s_{2}, \\
d_{n m}\left(c_{1}, c_{2}\right)=\iint_{\Omega} \varphi_{0}\left(s_{1}, s_{2}\right) \exp \left[i\left(c_{1} n s_{1}+c_{2} m s_{2}\right)\right] d s_{1} d s_{2},
\end{gathered}
$$

$$
d_{0}\left(c_{1}, c_{2}\right)=\iint_{\Omega} \Psi_{0}\left(s_{1}, s_{2}\right) \varphi_{0}\left(s_{1}, s_{2}, c_{1}, c_{2}\right) d s_{1} d s_{2} .
$$

For coefficients of the matrix $\mathbf{A}_{M}\left(c_{1}, c_{1}\right)=$ $\left\|a_{n m}^{(k l)}\left(c_{1}, c_{1}\right)\right\|_{\substack{k, n=-N \div N \\ m, l=-M \div M}}$ the equality $a_{m n}^{(l k)}\left(c_{1}, c_{1}\right)=$ $=\overline{a_{n m}^{(k l)}\left(c_{1}, c_{1}\right)}$ is valid, i.e. $\mathbf{A}_{M}$ is the Hermitian or self-adjoint matrix.

Write the equivalent to (26) nonlinear two-parametrical spectral problem, corresponding to a system of Equation (34), as

$$
\mathrm{A}_{M}\left(c_{1}, c_{2}\right) \mathbf{x} \equiv\left(\mathbf{E}_{M}-\mathbf{A}_{M}\left(c_{1}, c_{2}\right)\right) \mathbf{x}=0,
$$

where $\mathbf{E}_{M}$ is a unit matrix of dimension $N_{2} \times M_{2}$.

In order that the system (34) should have distinct from zero solutions, it is necessary

$$
\Psi\left(c_{1}, c_{2}\right)=\operatorname{det}\left(\mathbf{E}_{M}-\mathbf{A}_{M}\left(c_{1}, c_{2}\right)\right)=0 .
$$

It is easy to be convinced, that $\Psi\left(c_{1}, c_{2}\right)$ is a real function. Really as $\mathbf{T}_{M}\left(c_{1}, c_{2}\right)$ is the Hermitian matrix then it is obvious that $\left(\mathbf{E}-\mathbf{A}_{M}\left(c_{1}, c_{2}\right)\right)$ is also the Hermitian matrix. It is known [19] that the determinant of the Hermitian matrix is a real number. So, $\Psi\left(c_{1}, c_{2}\right)$ is a real function with respect to the real arguments $c_{1}$ and $c_{2}$.

Therefore, the problem on finding the set of eigenvalues of (25) or equivalent linear algebraic system (34) is reduced to finding the nulls of the function $\Psi\left(c_{1}, c_{2}\right)$.

Consider a necessary later on auxiliary one-dimensional spectral problem (as a special case of the problem (35)) on the ray $c_{2}=\gamma c_{1}$ ( $\gamma$ is a real coefficient, $\left.\left(c_{1}, c_{2}\right) \in \Lambda_{c}\right)$. Introduce into consideration the matrix function $\mathrm{A}_{M}{ }_{M}\left(c_{1}\right) \equiv \mathrm{A}_{M}\left(c_{1}, \gamma c_{1}\right)$ and connected with it the one-dimensional spectral problem

$$
\mathrm{A}_{M}{ }_{M}\left(c_{1}, \gamma c_{1}\right) \mathbf{x}=\left(\mathbf{E}_{M}-\mathbf{A}_{M}\left(c_{1}, \gamma c_{1}\right)\right) \mathbf{x}=0 .
$$

It is easy to be convinced, that from the properties of coefficients of matrix $\mathbf{A}_{M}\left(c_{1}, c_{2}\right)$ follows, that the matrix function $\mathrm{A}_{M}\left(c_{1}, c_{2}\right)$ is continuous and differentiable on the variables in any open and limited domain $\Lambda \subset \Lambda_{c} \subset{ }^{\circ 2}$. In other words $A_{M}\left(c_{1}, c_{2}\right)$ is a holomorphic matrix - function, if $c_{1}, c_{2}$ to continue into the domain of complex variables.

Corresponding to (37), Equation (36) has the form

$$
\Psi\left(c_{1}, \gamma c_{1}\right)=\operatorname{det}\left(\mathbf{E}_{M}-\mathbf{A}_{M}\left(c_{1}, \gamma c_{1}\right)\right)=0 .
$$

We denote the spectrums of the problems (35) and (37) as $s(\mathrm{~A})$ and $s(\mathrm{~A} \%$, respectively, and the parameter domain $c_{1}$ as $\Lambda_{c_{1}}=\left\{c_{1}: 0<c_{1} \leq a\right\}$. Then for proper- 
ties of the spectrum of (35) the Theorem 1 from [9] is applied which relatively to (35) is formulated thus:

Theorem 2. Let at each $\mathbf{c}=\left(c_{1}, c_{2}\right) \in \Lambda_{c}$ the matrix $\mathrm{A}_{M}\left(c_{1}, c_{2}\right) \in \mathrm{L}\left(\mathfrak{f}^{N_{2} \times M_{2}}, \mathfrak{£}^{N_{2} \times M_{2}}\right)$ be the Fredholm operator with a zero index, the matrix - function A $(\cdot, \cdot): \Lambda_{c} \rightarrow \mathrm{L}\left(\mathfrak{£}^{N_{2} \times M_{2}}, \AA^{N_{2} \times M_{2}}\right)$ be holomorphic in the domain $\Lambda_{c}$ and $s\left(\mathrm{~A} \% \neq \Lambda_{c_{1}}\right.$. Moreover, let function $\Psi\left(c_{1}, c_{2}\right)$ be continuously differentiable in $\Lambda_{c}$. Then:

1) Each point of a spectrum $c_{1}^{(0)} \in s(\mathrm{~A} \%$ is isolated and it is eigenvalue of the matrix - function $\mathrm{A}^{\%}\left(c_{1}\right) \equiv \mathrm{A}\left(c_{1}, \gamma c_{1}\right)$, to it is corresponding the finite-dimensional eigensubspace $\left.N\left(A^{\%} \% c_{1}^{(0)}\right)\right)$ and finite-dimensional root subspace;

2) Each point $\mathbf{c}^{(0)}=\left(c_{1}^{(0)}, \gamma c_{1}^{(0)}\right) \in \Lambda_{c}$ is a point of spectrum of the matrix - function $\mathrm{A}\left(\lambda_{1}, \lambda_{2}\right)$;

3) If $\Psi_{c_{2}}^{\prime}\left(c_{1}^{(0)}, c_{2}^{(0)}\right) \neq 0$ then in some vicinity of the point $c_{1}^{(0)}$ there is a unique continuous differentiable function $c_{2}=c_{2}\left(c_{1}\right)$ solving the Equation (36), i.e. in some bicircular domain $\Lambda_{0}=\left\{\left(c_{1}, c_{2}\right):\left|c_{1}-c_{1}^{(0)}\right|<\varepsilon_{1}\right.$, $\left.\left|c_{2}-c_{2}^{(0)}\right|<\varepsilon_{2}\right\}$ there exists a connected component of spectrum of the matrix-function $\mathrm{A}\left(c_{1}, c_{2}\right)$ ( where $\varepsilon_{1}$, $\varepsilon_{2}$ are small real constants).

Proof of this theorem concerning the nonlinear two-parametrical spectral problem of the type (35) for more general case (when the operators $E$ and $T\left(c_{1}\right.$, $c_{2}$ ) act in the infinite dimensional Banach space) is presented in [9]. For satisfaction of conditions of Theorem 1 from [9] it is necessary to show that the matrix function $\mathrm{A}\left(c_{1}, c_{2}\right)$ is the Fredholm matrix at $\left(c_{1}, c_{2}\right) \in \Lambda_{c}$. This property follows from the known equality [19] $\operatorname{dim}(\operatorname{kerA})=\operatorname{dim}(\operatorname{kerA} *)$.

The existence of connected components of spectrum of the matrix - function $\mathrm{A}\left(c_{1}, c_{2}\right)$, under condition of $\Psi_{c_{2}}^{\prime}\left(c_{1}^{(0)}, c_{2}^{(0)}\right) \neq 0$, follows from the existence theorem of implicitly given function [20, 21].

Let $c_{1}^{(i)}$ be a root of (38). Then $\left(c_{1}^{(i)}, c_{2}^{(i)}=\gamma c_{1}^{(i)}\right) \in \Lambda_{c}$ is eigenvalue of the problem (33). Consider the equation $\Psi\left(c_{1}, c_{2}\right)=0$ as a problem on finding the implicitly given function $c_{2}=c_{2}\left(c_{1}\right)$ in the vicinity of a point $c_{1}^{(i)}$ for which the conditions of existence theorem [21] are satisfied. Hence we have the Cauchy problem

$$
\begin{gathered}
\frac{d c_{2}}{d c_{1}}=-\frac{\Psi_{c_{1}}^{\prime}\left(c_{1}, c_{2}\right)}{\Psi_{c_{2}}^{\prime}\left(c_{1}, c_{2}\right)}, \\
c_{2}^{(i)}\left(c_{1}^{(i)}\right)=\gamma c_{1}^{(i)} .
\end{gathered}
$$

Solving numerically (39) and (40) in some vicinity of a point $c_{1}^{(i)}$, we find the $i$-th connected component of spectrum (spectral line) of the matrix - function $\mathrm{A}_{M}\left(c_{1}, c_{2}\right)$.

By found solutions of the Cauchy problem at the fixed values $\left(c_{1}^{(i)}, c_{2}^{(i)}\right)$ the eigenfunctions of (25) are determined through the eigenvectors of the matrix $\mathbf{A}_{M}\left(c_{1}^{(i)}, c_{2}^{(i)}\right)$ obtained by the known methods. Thus four-dimensional matrix $\quad \mathbf{A}_{M}$ is reduced to two-dimensional one by means of corresponding renumbering of elements.

\section{Numerical Algorithm of Finding the Solutions of a Nonlinear Equation}

Show one of iterative processes for numerical finding the solutions of the system (14) based on the successive approximations method [2]:

$$
\begin{aligned}
& u_{n+1}(Q)= B_{1}\left(u_{n}, v_{n}\right) \equiv \iint_{\Omega} K\left(Q, Q^{\prime}, \mathbf{c}\right) F\left(Q^{\prime}\right) \times \\
& \times \frac{u_{n}\left(Q^{\prime}\right)}{\sqrt{u_{n}^{2}\left(Q^{\prime}\right)+v_{n}^{2}\left(Q^{\prime}\right)}} d Q^{\prime}, \\
& v_{n+1}(Q)= B_{2}\left(u_{n}, v_{n}\right) \equiv \iint_{\Omega} K\left(Q, Q^{\prime}, \mathbf{c}\right) F\left(Q^{\prime}\right) \times \\
& \times \frac{v_{n}\left(Q^{\prime}\right)}{\sqrt{u_{n}^{2}\left(Q^{\prime}\right)+v_{n}^{2}\left(Q^{\prime}\right)}} d Q^{\prime} \quad(n=0,1, \ldots) .
\end{aligned}
$$

After substituting the function $\arg f_{n}(Q)=\operatorname{arctg}\left(v_{n}(Q)\right.$ $u_{n}(Q)$ ) (obtained on the basis of successive approximations (41)) into (12), we denote the obtained sequence of function values as $\left\{\mathbf{I}_{n}\right\}$. For the sequence $\left\{\mathbf{I}_{n}\right\}$ the Theorem 4.2.1 from [3] is fulfilled. From here follows, that the sequence $\left\{\mathbf{I}_{n}\right\}$ is a relaxation one for the functional (7) and numerical sequence $\left\{\sigma\left(\mathbf{I}_{n}\right)\right\}$ is convergent.

At realization of the iterative process (41) in the case of even on both arguments function $F\left(s_{1}, s_{2}\right)$ and symmetric domains $G$ and $\Omega$ it is expedient to use the property of invariance of integral operators $B_{1}(u, v)$, $B_{2}(u, v)$ in the system (14) concerning the type of parity of functions $u\left(s_{1}, s_{2}\right), v\left(s_{1}, s_{2}\right)$. The functions $u, v$ having certain type of evenness on corresponding arguments belong to the appropriate invariant sets $U_{i j}, V_{k 1}$ of the space $\mathbf{C}(\Omega)$. Here the indices $i, j, k, \mathrm{I}$ have 
values 0 or 1 . In particular, if $u\left(s_{1}, s_{2}\right) \in U_{01}$ then $u\left(-s_{1}, s_{2}\right)=u\left(s_{1}, s_{2}\right) \quad$ and $u\left(s_{1},-s_{2}\right)=-u\left(s_{1}, s_{2}\right)$. By direct check we are convinced that such inclusions take place:

$$
\begin{gathered}
B_{1}\left(U_{i j} \cup V_{k \mid}\right) \subset U_{i j}, \quad B_{2}\left(U_{i j} \cup V_{k \mid}\right) \subset V_{k \mid}, \\
\mathbf{B}\left(U_{i j} \cup V_{k \mid}\right) \subset U_{i j} \cup V_{k \mid} .
\end{gathered}
$$

The possibility of existence of fixed points of the operator $\mathbf{B}$ belonging to appropriate invariant set (i.e. solutions of system (14) and, respectively, Equation (10)) follows from these relations.

\section{Numerical Example}

Consider an example of approximation of the function $F\left(s_{1}, s_{2}\right)=\cos \left(\pi s_{1} / 2\right)\left|\sin \left(\pi s_{2}\right)\right|$ (Figure 1), given in the domain $\bar{G}=\left\{\left(s_{1}, s_{2}\right):\left|s_{1}\right| \leq 1,\left|s_{2}\right| \leq 1\right\} \subset \Omega$, for $N_{2} \times M_{2}=11 \times 11$ and values of parameters $c_{1}=1.6$ and $c_{2}=1.2$ belonging to the ray $c_{2}=0.75 c_{1}$. The possible branching lines of solutions of the system (14) and accordingly the Equation (10), as solutions of two-dimensional spectral problem (25), are shown in Figure 2. Here the first branching lines are denoted by numbers 1 and 2. To the solutions branching-off at the points of these lines there correspond the odd on $s_{2}$ functions $\arg f\left(s_{1}, s_{2}\right)$ and the coefficients of transformation $I_{n, m}(n=-N \div N, m=-M \div M)$ are real, but nonsymmetrical concerning to the plane $\mathrm{XOZ}$.

In Figure 3 in logarithmic scale are presented values of the functional $\sigma$ obtained on the solutions of two types at values of parameter $c_{2}=0.75 c_{1}$ : the curve 1

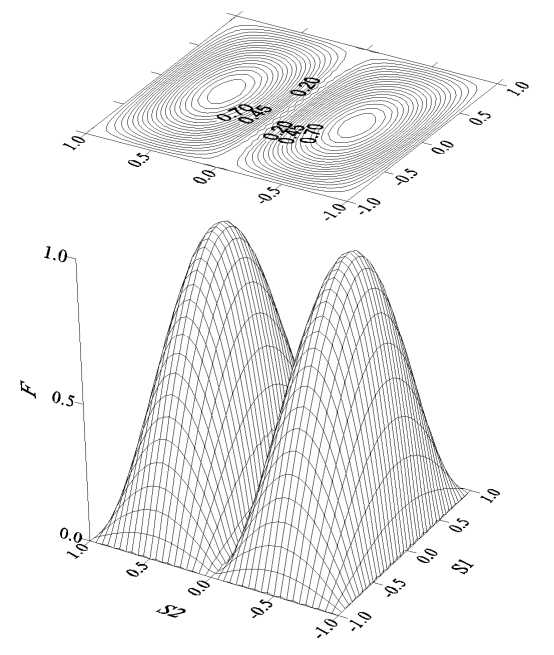

Figure 1. The function $F\left(s_{1}, s_{2}\right)=\cos \left(\pi s_{1} / 2\right)\left|\sin \left(\pi s_{2}\right)\right|$ given in the domain $\bar{G}=\left\{\left(s_{1}, s_{2}\right):\left|s_{1}\right| \leq 1,\left|s_{2}\right| \leq 1\right\} \subset \Omega$.

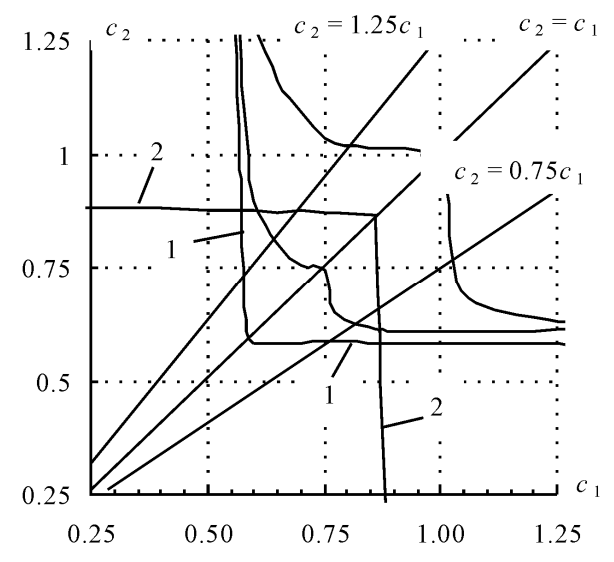

Figure 2. The branching lines of solutions

corresponds to solutions in a class of real functions $f_{0}(Q)$, curve 2 - to the branching-off solution with odd on $s_{2}$ argument $\arg f\left(s_{1}, s_{2}\right)$. From analysis of Figure 3 follows that at the point $c_{1} \approx 0.77$ from real solution branch-off more effective complex-conjugate between themselves solutions, on which the functional $\sigma$ accepts smaller values, than on the real solution. If to introduce into consideration parameter $C_{2}=M c_{2}$ characterizing the quantity of basic functions in transformation (1), the identical efficiency of approximation (identical values of the functional $\sigma$ on real and branching-off solutions) is reached with use of the branching-off solution at decrease of the quantity of basic functions on the value $\Delta C_{2}=0.75 \Delta c_{1}$.

An amplitude (a) and argument (b) of approximate function are given in Figure 4 for $c_{1}=1.6$ and $c_{2}=1.2$. The amplitude values of the Fourier Transform coefficients corresponding to this solution are shown in Figure 5. As we see in figure, the values of amplitudes of coefficients are nonsymmetrical concerning the plane

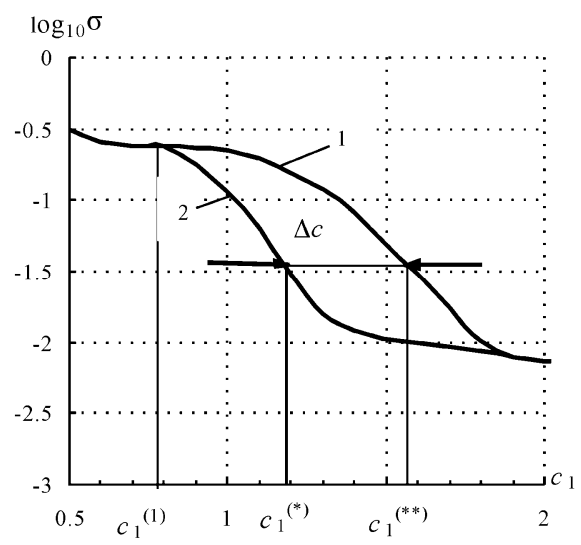

Figure 3. The values of functional on initial and branching-off solutions. 


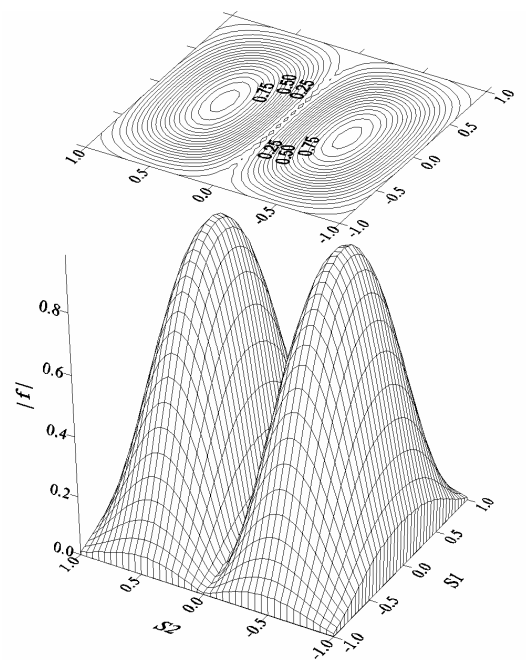

(a)

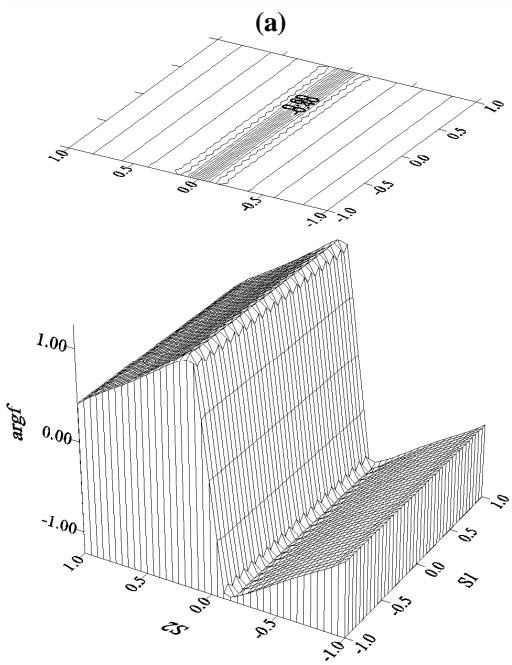

(b)

Figure 4. The modulus (a) and argument (b) of approximation function.

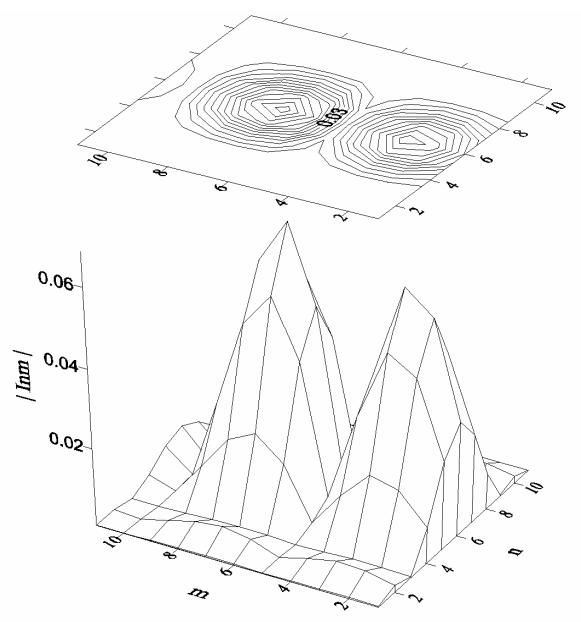

Figure 5. The optimum amplitude of Fouier transform coefficients.
$Y O Z$, but the amplitude of approximate function (Figure 4, a) is symmetric.

For comparison of approximate functions, corresponding to different solutions of (10), the curves corresponding to different types of the presented solutions in the section $s_{1} \equiv 0$ are given in Figure 6. The curve 1 corresponds to the given function $F\left(0, s_{2}\right)$, the curve 2 - to branching-off solution, the curve 3 - to real solution $f_{0}\left(0, s_{2}\right)$. Obviously that the branching-off solution better (in meaning of the functional $\sigma$ ) approaches the prescribed function by the module.

\section{Conclusion}

Mark the basic features and problems arising at investigation of the considered class of tasks:

The basic difficulty to solve this class of problems is study of nonuniqueness and branching of existing solutions dependent on the parameters $c_{1}, c_{2}$ entering into the discrete Fourier Transform.

As follows from investigations, presented, in particular, in [3,17] (for a special case, when $F\left(s_{1}, s_{2}\right)=$ $\left.F_{1}\left(s_{1}\right) \cdot F_{2}\left(s_{2}\right)\right)$, the quantity of the existing solutions grows considerably with increase of the parameters $c_{1}, c_{2}$. Let us indicate, that in many practical applications, in particular, in the synthesis problems of radiating systems, it is important to obtain the best approximation to the given function $F\left(s_{1}, s_{2}\right)$ at rather small values of parameters $c_{1}, c_{2}$. This allows limiting by investigation of several first points (lines) of branching.

To find the branching points (lines) of solutions of (8), it is necessary, as opposed to [3, 17], to solve not enough studied multiparametric spectral problem. The offered in this work approaches allow to find the solutions of a nonlinear two-parametric spectral problem for homogeneous integral equations with degenerate kernels analytically dependent on two spectral parameters.

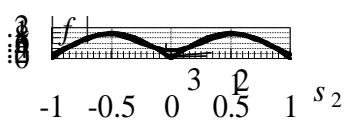

Figure 6. The given (curve 1) and approximation functions in the section $s_{2}=0$ corresponding to branching-off (curve 2) and real (curve 3) solutions. 
When finding the solutions to a system of Equation (14) by successive approximations method, to obtain the solutions of a certain type of parity of the function $\arg f\left(s_{1}, s_{2}\right)$ it is necessary to choose an initial approximation $\arg f_{0}\left(s_{1}, s_{2}\right)$ of the same type of parity according to (42).

To obtain the irrefragable answer concerning the branching-off solutions for certain values of parameters $c_{1}, c_{2}$ it is necessary to use the branching theory of solutions [18]. It is the object of special investigations.

\section{References}

[1] B. M. Minkovich and V. P. Jakovlev, "Theory of Synthesis of Antennas," Soviet Radio, Moscow, 1969.

[2] P. A. Savenko, "Numerical Solution of a Class of Nonlinear Problems in Synthesis of Radiating Systems," Computational Mathematics and Mathematical Physics, Vol. 40, No. 6, 2000, pp. 889-899.

[3] P. O. Savenko, "Nonlinear Problems of Radiating Systems Synthesis (Theory and Methods of the Solution)," Institute for Applied Problems in Mechanics and Mathematics, Lviv, 2002.

[4] G. M. Vainikko, "Analysis of Discretized Methods," Tartus Gos. University of Tartu, Tartu, 1976.

[5] R. D. Gregorieff and H. Jeggle, "Approximation von Eigevwertproblemen bei nichtlinearer Parameterabhängikeit," Manuscript Math, Vol. 10, No. 3, 1973, pp. 245271.

[6] O. Karma, "Approximation in Eigenvalue Problems for Holomorphic Fredholm Operator Functions I," Numerical Functional Analysis and Optimization, Vol. 17, No. 3-4, 1996, pp. 365-387.

[7] M. A. Aslanian and S. V. Kartyshev, "Updating of One Numerous Method of Solution of a Nonlinear Spectral Problem," Journal of Computational Mathematics and Mathematical Physics, Vol. 37, No. 5, 1998, pp. 713-717.
[8] S. I. Solov'yev, "Preconditioned Iterative Methods for a Class of Nonlinear Eigenvalue Problems," Linear Algebra and its Applications, Vol. 41, No. 1, 2006, pp. 210-229.

[9] P. A. Savenko and L. P. Protsakh, "Implicit Function Method in Solving a Two-dimensional Nonlinear Spectral Problem," Russian Mathematics (Izv. VUZ), Vol. 51, No. 11, 2007, pp. 40-43.

[10] V. A. Trenogin, "Functional Analysis," Nauka, Moscow ,1980.

[11] I. I. Privalov, "Introduction to the Theory of Functions of Complex Variables," Nauka, Moscow, 1984.

[12] A. N. Kolmogorov and S. V. Fomin, "Elements of Functions Theory and Functional Analysis," Nauka, Moscow, 1968.

[13] P. P. Zabreiko, A. I. Koshelev and M. A. Krasnoselskii, "Integral Equations," Nauka, Moscow, 1968.

[14] M. A. Krasnoselskii, G. M. Vainikko, and P. P. Zabreiko, "Approximate Solution of Operational Equations," Nauka, Moscow, 1969.

[15] I. I. Liashko, V. F. Yemelianow and A. K. Boyarchuk, "Bases of Classical and Modern Mathematical Analysis," Vysshaya Shkola Publishres, Kyiv, 1988.

[16] E. Zeidler, "Nonlinear Functional Analysis and Its Applications I: Fixed-Points Theorem," Springer-Verlag, New York, Berlin, Heidelberg, Tokyo, 1985.

[17] P. A. Savenko, "Synthesis of Linear Antenna Arrays by Given Amplitude Directivity Pattern," Izv. Vysch. uch. zaved. Radiophysics, Vol. 22, No. 12, 1979, pp. 1498-1504.

[18] M. M. Vainberg and V. A. Trenogin, "Theory of Branching of Solutions of Nonlinear Equations," Nauka, Moscow, 1969.

[19] V. V. Voyevodin and Y. J. Kuznetsov, "Matrices and Calculations," Nauka, Moscow, 1984.

[20] A. Gursa, "Course of Mathematical Analysis, Vol. 1, Part 1," Moscow-Leningrad, Gos. Technical Theory Izdat, 1933.

[21] V. I. Smirnov, "Course of High Mathematics, Vol. 1," Nauka, Moscow, 1965. 